\title{
Horst Wiesinger zum 80er
}

\author{
Karl Schwaha, Günter Holleis und Bruno Hribernik
}

Leoben, Österreich

Online publiziert 25. März 2020

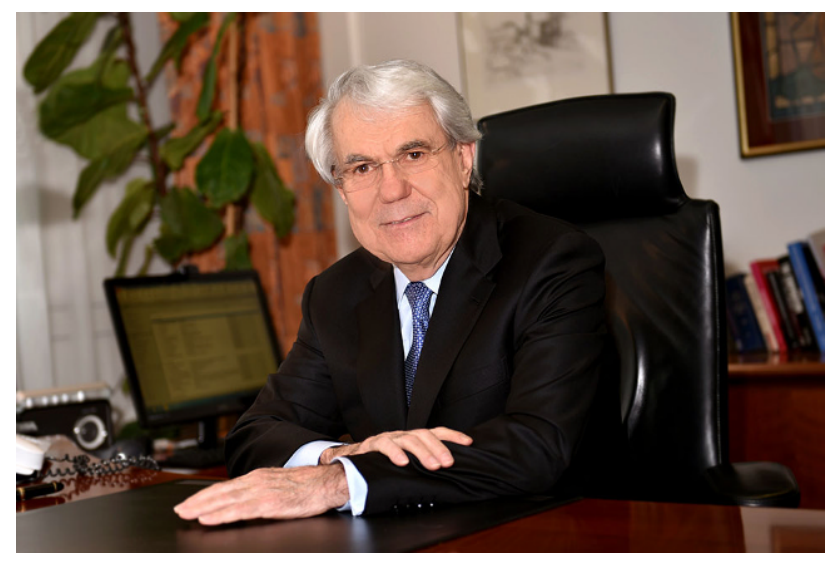

Abb. 1: Prof. Dr. Horst Wiesinger

Am Dienstag 24. März 2020 feiert Prof. Dr. Horst Wiesinger (Abb. 1) seinen achtzigsten Geburtstag. Seine berufliche Kariere war maßgeblich durch den Werkstoff Stahl beeinflusst, und als Mister Metallurgie ist er nach wie vor mit seinem Beratungsunternehmen Horst Wiesinger Consulting $\mathrm{GmbH}$ ein geschätzter Ansprechpartner in der internationalen Eisen- und Stahlindustrie.

Horst Wiesinger wurde am 24. März 1940 in Eferding geboren. Nach seiner Matura an der HTL in Linz arbeitete er als Konstrukteur beim Brückenbau der VOEST, entschied sich aber nach einem Jahr für ein Studium an der Montanistischen Hochschule in Leoben. Mit dem Abschluss des Studiums als Dipl. Ing. begann er 1966 seine berufliche Laufbahn im Industrieanlagenbau der VOEST-ALPINE auf dem Gebiet der Stranggießtechnik. Bereits in frühen Jahren wurde er als Resident Engineer zum Lizenzpartner in die USA entsandt. Dabei erkannte er, dass Innovation ein wesentlicher Erfolgsfaktor für den Anlagenbau ist und sein wird.

B. Hribernik ( $\square)$

A-8700 Leoben, Österreich

Bruno.Hribernik@asmet.at
Diese Erkenntnis begleitete und prägte ihn seine gesamte berufliche Laufbahn.

Im Jahr 1972 wurde er Abteilungsleiter der Projektierung von Stranggießanlagen. In der damals boomenden Stahlindustrie hat er maßgeblich zur erfolgreichen Markteinführung der eigenen Stranggießtechnik beigetragen, die neben der LD Stahlerzeugungstechnik ein bedeutsames Standbein des Metallurgieanlagenbaus wurde.

Im Jahr 1980 wurde sein Aufgabengebiet erweitert. Er wurde zum Direktor des Bereiches Stranggießanlagen, Walzwerke und Rohrerzeugung berufen, und in weiterer Folge erhielt er die Gesamtverantwortung für die Technologien vom Rohstoff bis zum Fertigprodukt.

Diese Periode war in der Stahlindustrie durch starke Fluktuation in der Produktion, geringes Wachstum und eine Änderung des Kundenverhaltens gekennzeichnet. Horst Wiesinger wurde bewusst, dass nicht mehr nur Lieferungen von Neuanlagen, sondern auch Problemlösungen über die gesamte Produktionskette und über alle EngineeringKompetenzen vom Markt gefordert wurden. Daraus ergaben sich neue Anforderungen an den Anlagenbau im Hinblick auf neue Dienstleistungen, Automatisierung und After Sales Service zur Effizienzsteigerung des Gesamtprozesses.

Im Jahr 1989 wurde Horst Wiesinger Mitglied des Vorstands der VOEST-ALPINE Industrieanlagenbau $\mathrm{GmbH}$ (VAI), dessen Vorsitz er 1995 übernahm. Unter seiner Führung erfolgte die Neuausrichtung des Unternehmens in Richtung Prozessentwicklung und daraus resultierend das Eingehen von Partnerschaften mit namhaften Stahlherstellern. Der Wandel in der Stahlindustrie erforderte auch einen Strukturwandel der Anlagenbauer und eine Internationalisierung mit dem Aufbau von lokalen Kompetenzen als Erfolgsfaktor.

Im Jahr 1998 erfolgte seine Berufung in den Vorstand des VATECH-Konzerns, dem er bis 2001 als GeneraldirektorStellvertreter angehörte.

In all seinen Funktionen gab Horst Wiesinger zahlreiche neue und entscheidende Impulse in Richtung Innovation und Wettbewerbsfähigkeit. Er initiierte und förderte die Entwicklung von Technologien zur Schmelz- und Direktre- 
duktion (COREX, FINEX), zur Decarbonisierung durch Wasserstoffmetallurgie und zum Recycling mit dem Ziel, mit den „Best available environmental technologies" ein Zero Waste Hüttenwerk anbieten zu können. Insbesondere war ihm auch die Maximierung des Einsatzes des Sekundärrohstoffs Schrott ein großes Anliegen.

Im Jahr 2000 schloss Horst Wiesinger ein Doktoratsstudium an der Montanuniversität Leoben ab. In seiner Doktorarbeit hat er bereits visionär die Bedeutung des Wasserstoffs für die Metallurgie der Zukunft hervorgehoben.

Im Jahr 2001 machte sich Horst Wiesinger selbständig und gründete sein Beratungsunternehmen Horst Wiesinger Consulting (HWC), das bis heute mit einem Netzwerk von Experten erfolgreich Studien, Ausschreibungen, Planungen, Konzepterstellungen und Betriebsassistenz für die Eisen- und Stahlindustrie anbietet.

Darüber hinaus bekleidete Horst Wiesinger mehrere Aufsichtsratsmandate in nationalen und internationalen Industrieunternehmen und engagierte sich als stellvertretender Vorsitzender des Rates für Forschung und Entwicklung des Landes Oberösterreich bei der Gestaltung von Zukunftskonzepten in den Bereichen Forschung und Technologieentwicklung.

Die herausragenden Leistungen von Horst Wiesinger wurden auch mit zahlreichen Ehrungen und Auszeichnungen gewürdigt. Hervorzuheben sind:

- der "Willy Korf Steel Vision Award“ der American Metal Society

- die Verleihung des Titels „Bergrat“ durch die Montanuniversität Leoben

- die Ernennung zum Mitglied der russischen Akademie der Ingenieurwissenschaften

- die Verleihung der großen Goldenen Medaille der Stadt Linz
- die Verleihung der Ehrenbürgerschaft der Montanuniversität Leoben und

- die Verleihung des Ehrentitels "Professor" durch den Bundespräsidenten

Besonders betont werden soll an dieser Stelle die Rolle von Horst Wiesinger bei der Neuausrichtung der Eisenhütte Österreich, d. h. Gründung der ASMET, vor zwanzig Jahren. Er hat dabei nicht nur in seiner damaligen Funktion als Vereinsvorstandsmitglied konzeptiv mitgewirkt, sondern auch als Vorsitzender des Vorstands der VOEST-ALPINE Industrieanlagenbau $\mathrm{GmbH}$ (VAI) und durch seine guten Kontakte zu anderen Industriefirmen wesentlich dazu beigetragen, die Wege zu ebnen und die Mittel für die Startphase der ASMET einzuwerben. Die Gründung der ASMET war gleichzeitig auch eine Initialzündung für die strategischen Kooperationen wichtiger Firmen im Bereich der Stahl-, Metallherstellung und Verarbeitung, dem Metallurgieanlagenbau sowie deren Zulieferanten in Österreich und ein entscheidender Schritt zur Erhöhung der internationalen Sichtbarkeit der österreichischen Industrie und insbesondere der Forschung am Sektor Material und Metallurgie.

Horst Wiesinger ist sicherlich ein außergewöhnlicher Sonderfall für aktives Altern, denn ein Ende seiner Aktivitäten zeichnet sich nicht ab. Mit ungebrochenem Enthusiasmus ist er weltweit in Sachen Stahl unterwegs.

Wir wünschen Dir, lieber Horst, noch viele schöne Jahre und vor allem Gesundheit.

Glückauf!

Karl Schwaha

Günter Holleis

Bruno Hribernik 